\title{
Factorial design to obtain magnetized poly(ethyl acrylate-co-divinylbenzene)
}

\author{
Kelly Lúcia Nazareth Pinho de Aguiar ${ }^{1 *}$ (D), Kaio Alves Brayner Pereira ${ }^{1}$, Marcelo Sierpe Pedrosa $^{1}$ and \\ Márcia Angélica Fernandes e Silva Neves ${ }^{1}$
}

\author{
1'Instituto Federal de Educação, Ciência e Tecnologia do Rio de Janeiro - IFRJ, Nilópolis, RJ, Brasil \\ *kelly.pinhoo@gmail.com
}

\begin{abstract}
Magnetized polymers are produced by incorporating magnetic particles in a polymeric matrix. This article describes the use of the suspension polymerization technique using ethyl acrylate and divinylbenzene as monomers, in the presence of heptane and/or toluene as diluent, initiated by free radicals. To produce the polymer, we first performed fractional factorial planning to help visualize the factors that could influence the results, to verify the action of different responses simultaneously. Five factors were evaluated that influence the production of the polymer and incorporation of iron in the matrix. Infrared spectroscopy, X-ray fluorescence, magnetic force testing and scanning electron microscopy were used to characterize the samples. The results indicated the positive influence of the quantity of the polymerization initiator on the yield of the process and the negative effect of the content of divinylbenzene on the incorporation of iron in the matrix and on the magnetic force.
\end{abstract}

Keywords: magnetized polymer, factorial design, suspension polymerization.

How to cite: Aguiar, K. L. N. P., Pereira, K. A. B., Pedrosa, M. S., \& Neves, M. A. F. S. (2019). Factorial design to obtain magnetized poly(ethyl acrylate-co-divinylbenzene). Polímeros: Ciência e Tecnologia, 29(2), e2019020. https:// doi.org/10.1590/0104-1428.04118

\section{Introduction}

Magnetized polymer spheres, also called magnetized resins, are obtained by incorporating magnetic particles in a polymeric resin. These resins have attracted strong interest in the field of research and development, due to their excellent potential for application in environmental remediation, to remove pollutants from affected sites. After their functionalization, they can act by adsorbing a contaminant and then be removed by filtration or application of an external magnet ${ }^{[1]}$.

These structures can be produced by various polymerization techniques. Suspension polymerization is the most common to generate magnetic spheres, due to the facility of separating the product from the reaction medium, the low level of impurities and the consistency of the spherical particles produced $^{[2,3]}$.

Because of the large number of factors that can influence the process of making polymers, and consequently the diverse characteristics of the resulting products, it is very important to plan the experiments to obtain the largest quantity of relevant data with the smallest number of reactions possible. Among the types of experimental optimization, a multivariate method called factorial planning stands out, whereby it is possible to evaluate the responses of two or more variables simultaneously and determine which are most relevant ${ }^{[4,5]}$.

The synthesis of magnetic microspheres using monomers such as methyl methacrylate crosslinked with divinylbenzene has been reported ${ }^{[6-9]}$. To date, no results of magnetized resins have been found with the use of ethyl acrylate monomer and showing the influence of the experimental factors. This article describes the production of a magnetized form of the resin poly(ethyl acrylate-co-divinylbenzene) and evaluates the factors that influence the main characteristics of this polymer, by applying a factorial experimental design with five factors, to determine the more relevant experimental factors in the characteristics of the material for further optimization.

The structural characteristics were assessed by infrared spectroscopy, while the morphology was evaluated by scanning electron microscopy. The content of iron incorporated was detected by the X-ray fluorescence technique. Finally, the magnetic force was measured by a method developed by the Laboratory of Biopolymers and Sensors of the Institute of Macromolecules of Rio de Janeiro Federal University (LaBioS/IMA-UFRJ).

\section{Materials and Methods for Preparation of Magnetized Poly(ethyl acrylate-co-divinylbenzene)}

\subsection{Materials}

Divinylbenzene (DVB) (Nitriflex S/A, Brazil) was washed with $5 \%$ aqueous sodium hydroxide to remove inhibitors. Ethyl acrylate (Vetta Química LTDA, Brazil), benzoyl peroxide (BPO) (Vetec Química Fina LTDA, Brazil), toluene (TOL) (Vetec Química Fina LTDA, Brazil), heptane (HEP) 
(Vetec Química Fina LTDA, Brazil), glycerin (bidistilled glycerin, USP 99.5\%, purchased from Audaz), poly(vinyl alcohol) (PVA) (Mowiol ${ }^{\circledR}$ 18-88 from Sigma-Aldrich, Brazil) and maghemite (particle size $=12 \mathrm{~nm}$ ) were used as received.

\subsection{Preparation of the magnetized poly(ethyl acrylate-co-divinylbenzene)}

The magnetized poly(ethyl acrylate-co-divinylbenzene) was obtained by suspension polymerization, using a Syrris Atlas Sodium reactor with temperature control and overhead stirring. The continuous medium was composed of water or a mixture of water and glycerin (1:1 or 3:1), and the suspension agent was PVA, with variable concentrations as established in the experimental factorial design. This phase was kept under magnetic stirring at $70{ }^{\circ} \mathrm{C}$ until total dissolution of the suspension agent. The total volume of the continuous medium was four times that of the organic phase.

The organic phase was prepared in a round-bottom flask in the Atlas Sodium system by adding ethyl acrylate, DVB as crosslinker, varying the concentration from 20 to $40 \%$ by molar mass, and BPO as polymerization initiator, varying its concentration from $1 \%$ to $10 \%$ in relation to the total number of mols of the monomer. The diluent was TOL, HEP or a mixture of TOL:HEP in 1:1 proportion, while maghemite ( $5 \%$ by mass of the monomers) was used to incorporate iron in the polymeric matrix. The quantities used to prepare the organic phase were determined in the experimental factorial design, reported in Table 1, where the total amount of monomers employed was $0.1 \mathrm{~mol}$. This phase was kept under stirring at $400 \mathrm{rpm}$ and $50^{\circ} \mathrm{C}$ for one hour, to improve the affinity of the maghemite with the organic phase and enable greater incorporation of iron in the polymer produced.

The aqueous phase was added to the round-bottom flask containing the organic phase and the temperature of the system was increased to $70^{\circ} \mathrm{C}$ while maintaining magnetic stirring of $400 \mathrm{rpm}$. The polymerization in suspension lasted 24 hours.

At the end of the process, the poly(ethyl acrylateco-divinylbenzene) was removed from the flask and purified with water, ethanol and methanol.

\subsection{Experimental factorial design}

Fractional factorial planning was carried out to ascertain the most important variables to maximize the yield, iron content and magnetic force of the poly(acrylate-co-divinylbenzene) obtained by the suspension polymerization in the presence of maghemite.

In this experimental design, the influence of five factors was evaluated, namely: percentages of poly(vinyl alcohol) (PVA), divinylbenzene (DVB), benzoyl peroxide (BPO), diluents and the composition of the continuous medium. With these factors, the number of experiments was calculated according to Equation 1: Calculation of the number of experiments in the fractional design ${ }^{[10]}$.

$$
2^{\mathrm{n}-1}=2^{5-1}=2^{4}=16
$$

For better reliability of the method, we used the central point condition in triplicate, for a total of 19 experiments. The experimental conditions of each polymerization reaction are reported in Table 1. The factorial design described in this work was created by the Design-Ease $10^{\circledR}$ software, to obtain the following response data: yield, iron content and magnetic force.

The fractional factorial design used in this work can confound some effects at a lower hierarchical level (confounding or aliasing), as shown in Table 2. Therefore, it was necessary to evaluate the primary effects to verify if

Table 1. Factorial design to produce poly(ethyl acrylate-co-divinylbenzene).

\begin{tabular}{|c|c|c|c|c|c|}
\hline CODE & $\begin{array}{c}A=\text { PVA } \\
(\%)\end{array}$ & $\begin{array}{c}B=\text { DVB } \\
(\%)\end{array}$ & $\begin{array}{c}\mathrm{C}=\mathrm{BPO} \\
(\%)\end{array}$ & $\begin{array}{c}D=\text { HEP } / \text { TOL } \\
(\%)\end{array}$ & $\begin{array}{c}\mathrm{E}=\mathrm{GLYC} / \text { Water } \\
(\%)\end{array}$ \\
\hline R1 & 0.5 & 20 & 1 & $0 / 100$ & $50 / 50$ \\
\hline $\mathrm{R} 2$ & 5 & 20 & 1 & $100 / 0$ & $50 / 50$ \\
\hline R3 & 0.5 & 20 & 10 & $100 / 0$ & $50 / 50$ \\
\hline R4 & 0.5 & 40 & 1 & $0 / 100$ & $0 / 100$ \\
\hline R5 & 5 & 40 & 10 & $100 / 0$ & $50 / 50$ \\
\hline R6 & 0.5 & 40 & 1 & $100 / 0$ & $50 / 50$ \\
\hline R7 & 5 & 20 & 10 & $100 / 0$ & $0 / 100$ \\
\hline $\mathrm{R} 8$ & 5 & 20 & 1 & $0 / 100$ & $0 / 100$ \\
\hline R9 & 0.5 & 40 & 10 & $0 / 100$ & $50 / 50$ \\
\hline R10 & 5 & 40 & 1 & $100 / 0$ & $0 / 100$ \\
\hline R11 & 5 & 40 & 1 & $0 / 100$ & $50 / 50$ \\
\hline R12 & 2.75 & 30 & 5.5 & $50 / 50$ & $25 / 75$ \\
\hline R13 & 5 & 40 & 10 & $0 / 100$ & $0 / 100$ \\
\hline R14 & 0.5 & 40 & 10 & $100 / 0$ & $0 / 100$ \\
\hline R15 & 0.5 & 20 & 10 & $0 / 100$ & $0 / 100$ \\
\hline R16 & 0.5 & 20 & 1 & $100 / 0$ & $0 / 100$ \\
\hline R17 & 5 & 20 & 10 & $0 / 100$ & $50 / 50$ \\
\hline R18 & 2.75 & 30 & 5.5 & $50 / 50$ & $25 / 75$ \\
\hline R19 & 2.75 & 30 & 5.5 & $50 / 50$ & $25 / 75$ \\
\hline
\end{tabular}

PVA = Poly(vinyl alcohol); DVB = Divinylbenzene; BPO = Benzoyl peroxide; HEP/TOL = Heptane/Toluene; GLYC = Glycerin. 
Table 2. Possible aliasing of the fractional factorial design.

\begin{tabular}{cc}
\hline Effects & Aliasing effect* \\
\hline $\mathrm{A}$ & $\mathrm{A}$ \\
$\mathrm{B}$ & $\mathrm{B}$ \\
$\mathrm{C}$ & $\mathrm{C}$ \\
$\mathrm{D}$ & $\mathrm{D}$ \\
$\mathrm{E}$ & $\mathrm{E}$ \\
$\mathrm{AB}$ & $\mathrm{AB}+\mathrm{CDE}$ \\
$\mathrm{AC}$ & $\mathrm{AC}+\mathrm{BDE}$ \\
$\mathrm{AD}$ & $\mathrm{AD}+\mathrm{BCE}$ \\
$\mathrm{AE}$ & $\mathrm{AE}+\mathrm{BCD}$ \\
$\mathrm{BC}$ & $\mathrm{BC}+\mathrm{ADE}$ \\
$\mathrm{BD}$ & $\mathrm{BD}+\mathrm{ACE}$ \\
$\mathrm{BE}$ & $\mathrm{BE}+\mathrm{ACD}$ \\
$\mathrm{CD}$ & $\mathrm{CD}+\mathrm{ABE}$ \\
$\mathrm{CE}$ & $\mathrm{CE}+\mathrm{ABD}$ \\
$\mathrm{DE}$ & $\mathrm{DE}+\mathrm{ABC}$ \\
\hline
\end{tabular}

*Some parameters cannot be estimated independently.

any of these alone were significant, and then to ascertain the correct synergistic effect.

The application of a factorial design allows visualizing the positive and negative, primary and synergistic effects that influence the responses under analysis. To allow better visualization of these results, we used Pareto charts, where each factor is quantified according to its contribution to the problem and ranked in decreasing order of influence, to enable identification of the most important effects ${ }^{[10]}$.

To evaluate the statistical significance of the results, we calculated the p-value, also called the descriptive level of p-level, which estimates the degree of the null hypothesis $\left(\mathrm{H}_{\mathrm{o}}\right)$ for a model to be discarded ${ }^{[11,12]}$. It can thus be considered a reliability index of the model obtained. The p-value depends directly on a determined sample, supplying a measure of the strength of the results obtained by the test, in comparison with simple rejection or not of the null hypothesis. In statistics, this type of hypothesis generally states there is no relationship between two measured phenomena ${ }^{[13]}$. Technically speaking, the smaller the p-value of a determined result is, the stronger the evidence is against the null hypothesis, making the data obtained more reliable in indicating that the relationship is true between the parameters analyzed in the sample and the results representing the entire population ${ }^{[12]}$. In this framework, p-values lower than 0.01 are considered very reliable, while $0.01 \leq \mathrm{p}<0.05$ indicates moderate reliability, $0.05 \leq p<0.10$ denotes weak reliability, and $p \geq 0.10$ is considered not significant ${ }^{[5]}$.

\subsection{Characterization}

Fourier-transform infrared spectroscopy (FTIR) was used for structural analysis of the polymers, using a Nicolet iS5 spectroscope from Thermo Fisher Scientific, with potassium bromide $(\mathrm{KBr})$ pellets, 32 scans per sample and $4 \mathrm{~cm}^{-1}$ resolution. Scanning electron microscopy was employed to study the morphological characteristics, using a Phenom ProX SEM with a magnetic particle sample holder, setting the acceleration voltage to $10 \mathrm{kV}$. A Rigaku REX CG energy dispersive X-ray fluorescence spectrometer was used to quantify the iron incorporated in the polymeric matrix. Finally, the magnetic force was measured with an apparatus developed by the research group of the LaBioS/IMA-UFRJ, using a method to determine the magnetic force in function of the magnetic field generated in the sample. This system is composed of an electromagnet; a Teflon sample holder with volume of $1.76 \mathrm{~cm}^{3}$; a Shimadzu AY22 analytical balance; an ICEL PS4100 power source; a TLMP-HALL-02 Gaussmeter; and an ICEL MD-6450 ammeter ${ }^{[2]}$.

\section{Results and Discussion}

The polymers obtained from copolymerization of ethyl acrylate and divinylbenzene were characterized by FTIR. Since all the polymers produced presented the same spectrum only one of them is shown here, in Figure 1.

By reference against the bands characteristic of the infrared absorption of the groups present in the poly(ethyl acrylate-co-divinylbenzene), it was possible to analyze the bands depicted in Figure 1 and characterize the product obtained from the polymerization process. The band at $2977 \mathrm{~cm}^{-1}$ denotes the $\mathrm{C}-\mathrm{H}$ axial deformation of aromatics, and is partly superimposed on the $\mathrm{C}-\mathrm{H}$ axial deformation band of the aliphatics at $2932 \mathrm{~cm}^{-1}$. The band at $1734 \mathrm{~cm}^{-1}$ can be attributed to the $\mathrm{C}=0$ axial deformation of ester, while the bands at $1604 \mathrm{~cm}^{-1}, 1473 \mathrm{~cm}^{-1}$ and $1448 \mathrm{~cm}^{-1}$ denote the $\mathrm{C}=\mathrm{C}$ axial deformations of aromatic nuclei and the band at $1155 \mathrm{~cm}^{-1}$ is characteristic of the $\mathrm{C}-\mathrm{O}$ axial deformation of ester ${ }^{[14]}$.

Figure 2 presents the scanning electron microscopic images with 1000x magnification, where the bright white parts are the iron particles incorporated in the polymeric matrix.

The SEM images reveal the morphology of the magnetized resins, which did not exhibit a particular pattern. Some of the particles were spherical, others were fragmented and some were grouped in large agglomerates. These observations can be explained by the PVA content. It was verified that when a low PVA content is used, there is no suspension stability, forming brittle particles (Figure 2c). On the other hand, a high content of PVA provided the formation of agglomerate (Figure 2a and 2b). The center point of PVA content showed the best stabilization. These micrographs represent all the resins synthesized in this work.

The yields, iron content incorporated and magnetic force obtained from the polymerization reactions are reported in Table 3.

\subsection{Yields}

The Pareto chart obtained from the data on the polymerization yield (Table 3 ) revealed the most important factors regarding the response to the parameters, as seen in Figure 3.

The most important factor for the yield from the polymerization of poly(ethyl acrylate-co-divinylbenzene) was the quantity of initiator used in the process. Since this effect was positive, we can conclude that the yield increased with higher concentration of BPO in the reaction medium. With large quantities of BPO, thermal decomposition generates large quantities of free radicals, which attack the monomer 
Aguiar, K. L. N. P., Pereira, K. A. B., Pedrosa, M. S., \& Neves, M. A. F. S.

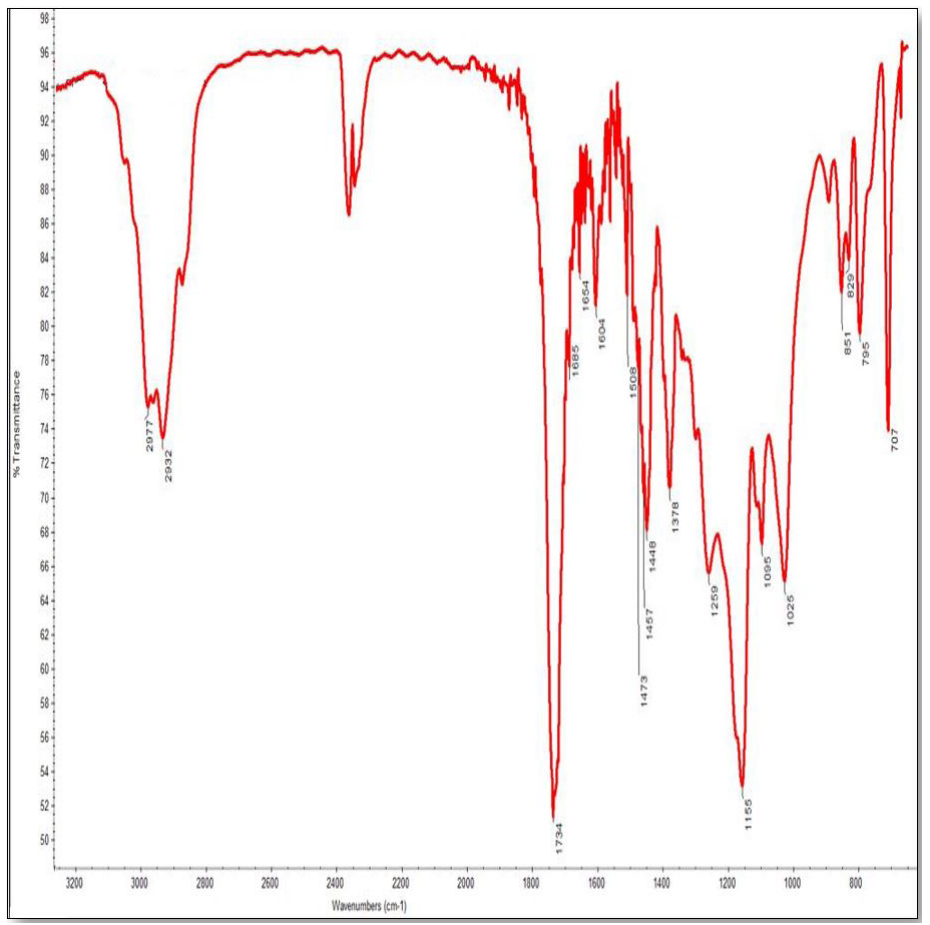

Figure 1. FTIR spectrum of poly(ethyl acrylate-co-divinylbenzene).

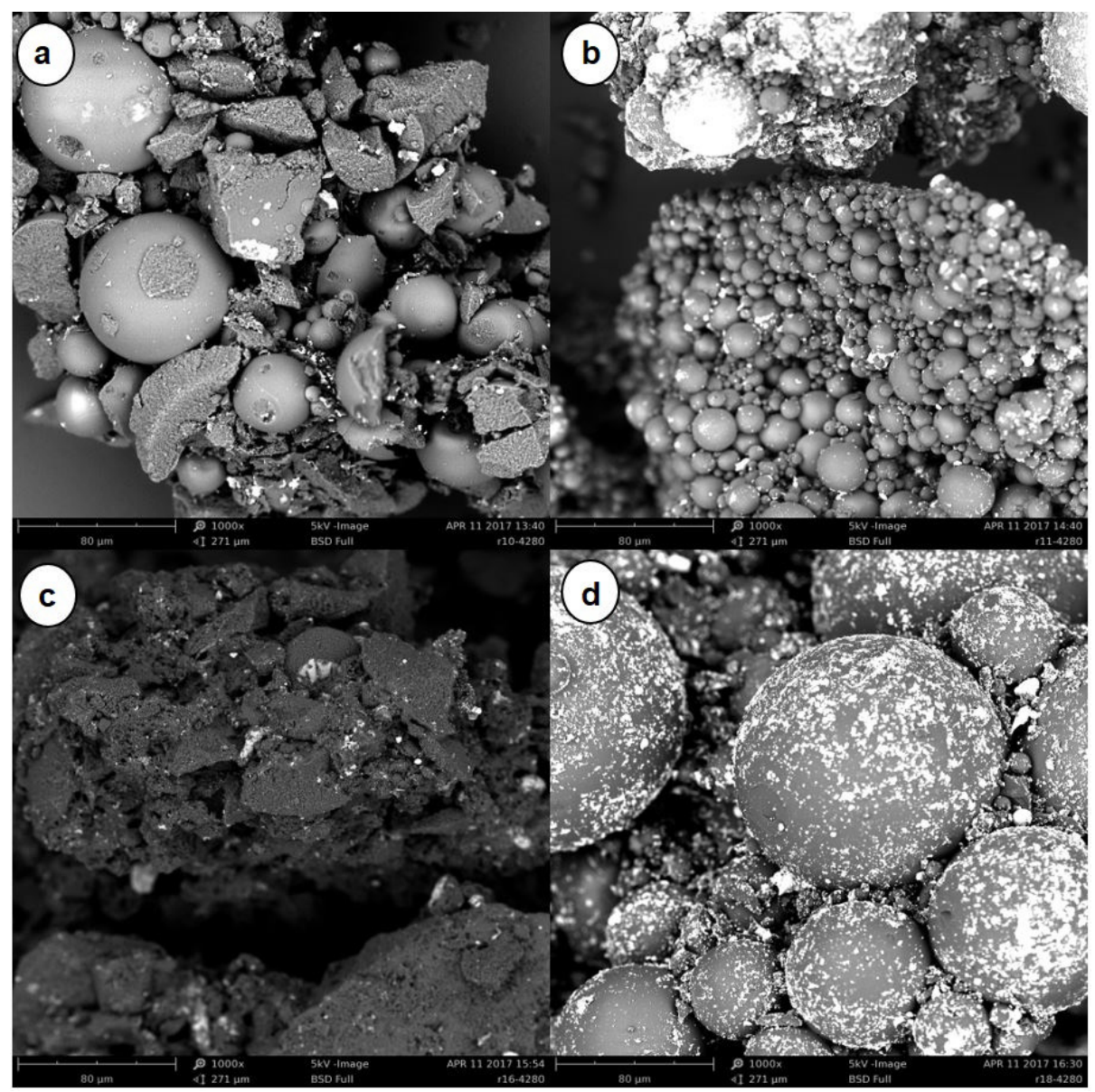

Figure 2. (a) R10; (b) R11; (c) R16; (d) R18. 
molecules, breaking the double bonds and starting the polymerization reaction. Therefore, the reaction is completed in less time due to the larger number of initiator species at the start of the polymerization process, thus increasing resin yield. As explained by Coutinho and Oliveira, the reaction kinetics shows that the polymerization speed is directly proportional to the concentration of monomers and the square root of the initiator concentration ${ }^{[15]}$.

Figure 3 shows that the second leading factor for yield of the process is $\mathrm{AD}$ synergy, i.e., the association of the suspension agent (PVA) and the diluent (heptane), acting

Table 3. Yields, iron content incorporated and magnetic force obtained from the polymerization reactions.

\begin{tabular}{cccc}
\hline Code & $\begin{array}{c}\text { Yield } \\
\text { (\%) }\end{array}$ & $\begin{array}{c}\text { Iron Content } \\
\text { (\% weight) }\end{array}$ & $\begin{array}{c}\text { Magnetic } \\
\text { Force (G/A) }\end{array}$ \\
\hline R1 & 77.3 & $1.280 \pm 0.000$ & 18.01 \\
R2 & 66.9 & $0.916 \pm 0.001$ & 18.99 \\
R3 & 95.9 & $1.980 \pm 0.000$ & 22.50 \\
R4 & 68.2 & $0.165 \pm 0.001$ & 4.35 \\
R5 & 95.1 & $0.589 \pm 0.001$ & 12.74 \\
R6 & 79.3 & $0.560 \pm 0.001$ & 5.06 \\
R7 & 82.9 & $1.187 \pm 0.006$ & 11.02 \\
R8 & 81.4 & $1.117 \pm 0.006$ & 20.28 \\
R9 & 96.1 & $0.998 \pm 0.003$ & 15.89 \\
R10 & 81.0 & $0.392 \pm 0.001$ & 14.99 \\
R11 & 83.6 & $0.244 \pm 0.001$ & 12.66 \\
R12 & 94.0 & $1.903 \pm 0.006$ & 15.42 \\
R13 & 96.5 & $0.838 \pm 0.001$ & 14.52 \\
R14 & 98.8 & $1.600 \pm 0.000$ & 2.55 \\
R15 & 76.4 & $0.402 \pm 0.002$ & 4.83 \\
R16 & 83.8 & $1.987 \pm 0.006$ & 12.14 \\
R17 & 88.9 & $1.670 \pm 0.000$ & 16.39 \\
R18 & 91.4 & $1.210 \pm 0.000$ & 10.07 \\
R19 & 92.4 & $1.370 \pm 0.000$ & 16.33 \\
\hline
\end{tabular}

$\mathrm{G} / \mathrm{A}=$ Gauss/Ampere.

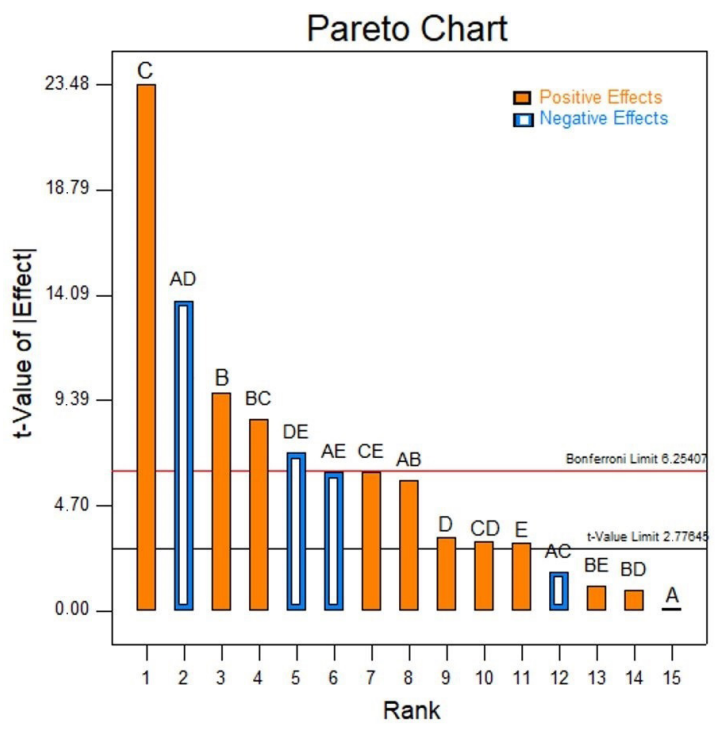

Figure 3. Pareto chart of yield data. negatively on the yield, but the primary effects A and D do not have significant influences, leading to the conclusion of confounding with the alias BCE, i.e., the synergy among the crosslinker DVB (B), the polymerization initiator BPO (C) and the addition of glycerin in the continuous medium $(\mathrm{E})$, since the primary parameters $\mathrm{B}$ and $\mathrm{C}$ are highly significant. The associated action of these three factors can be observed and better understood in Figure 4.

Figure 4 shows that the yield tends to rise with higher concentrations of BPO and DVB with the use of a continuous medium formed by $50 \%$ glycerin, revealing the synergistic effect of these three factors. In this situation, the polymerization reaction appears to start faster and the polymer formed seems to contain more crosslinks, so that glycerin molecules can be trapped in the crosslinks, increasing the mass of the poly(ethyl acrylate-co-divinylbenzene), and thus the yield of the suspension polymerization. However, this hypothesis requires additional analyses for confirmation.

The third most important factor for the yield is the concentration of DVB, with a positive effect for this response. This behavior can be explained by the increase of the crosslinks in the polymer chain, forming more resistant resins, making the particles more integral and avoiding loss of the polymer in the purification step.

The last factor with a significant contribution is the synergy between the $\mathrm{BC}$ factors, i.e., the cooperative action between the quantity of crosslinker (DVB) and the polymerization initiator (BPO), with a positive effect, Therefore, larger quantities of DVB and BPO also make the yield increase.

The contribution generated by each factor expressed as a percentage was also estimated. Only the factors $\mathrm{C}, \mathrm{BCE}$, $\mathrm{B}$ and $\mathrm{BC}$ made significant contributions, respectively of $43.95 \%, 15.27 \%, 7.51 \%$ and $5.82 \%$. These parameters with strong influence on the results obtained have a good level of significance, with $\mathrm{C}$ and $\mathrm{BCE}$ being very reliable, $\mathrm{B}$ reliable, and $\mathrm{BC}$ relatively less reliable (Table 4).

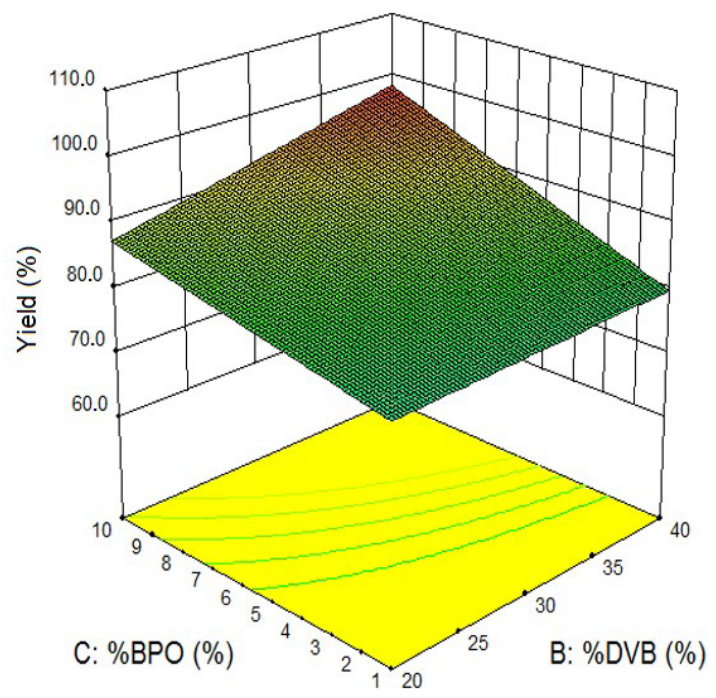

Figure 4. 3D surface for the influence of the alias BCE (Divinylbenzene, Benzoyl peroxide and Glycerin) on the yield of poly(ethyl acrylate-co-divinylbenzene). 
Table 4. Percentage of contribution of the effects that influence the polymerization yield.

\begin{tabular}{lccc}
\hline \multicolumn{1}{c}{ Factor } & Effect & \% Contribution & P-value* \\
\hline A - \% PVA & 0.062 & $9.232 \mathrm{E}-004$ & - \\
B - \%DVB & 5.64 & $\mathbf{7 . 5 1}$ & $\mathbf{0 . 0 3 7 1}$ \\
C - \%BPO & 13.64 & $\mathbf{4 3 . 9 5}$ & $\mathbf{0 . 0 0 0 1}$ \\
D -\%Heptane & 1.91 & 0.86 & - \\
E - \%Glycerin & 1.76 & 0.73 & - \\
AB & 3.39 & 2.71 & 0.1821 \\
AC & -1.01 & 0.24 & - \\
AD (alias BCE) & -8.04 & $\mathbf{1 5 . 2 7}$ & $\mathbf{0 . 0 0 6 2}$ \\
AE & -3.59 & 3.04 & 0.1596 \\
BC & 4.96 & $\mathbf{5 . 8 2}$ & $\mathbf{0 . 0 6 1 0}$ \\
BD & 0.54 & 0.068 & - \\
BE & 0.64 & 0.096 & - \\
CD & 1.79 & 0.76 & - \\
CE & 3.59 & 3.04 & 0.1596 \\
DE & -4.09 & 3.95 & 0.1137 \\
\hline
\end{tabular}

${ }^{*} \mathrm{P}$-value $=\mathrm{P}$-value is considered a reliability index of the model obtained, thus $\mathrm{p}$-values lower than 0.01 are considered very reliable, while $0.01 \leq \mathrm{p}<0.05$ indicates moderate reliability, $0.05 \leq \mathrm{p}<0.10$ denotes weak reliability and $\mathrm{p} \geq 0.10$ is considered not significant; $\mathrm{PVA}=$ Poly (vinyl alcohol); DVB = Divinylbenzene; $\mathrm{BPO}=$ Benzoyl peroxide; $\mathrm{BCE}=$ synergy among Divinylbenzene (B), Benzoyl peroxide $(\mathrm{C})$ and Glycerin $(\mathrm{E})$.

\subsection{Iron content in the magnetized resins}

$\mathrm{X}$-ray fluorescence was used to evaluate this parameter, where each sample was analyzed in triplicate, to obtain the mean of the three values, expressed as a percentage by weight. The results are reported in Table 3.

For this response, the effect with the greatest influence on the iron incorporation in the polymer matrix is factor $B$, i.e., the concentration of divinylbenzene used in the polymerization. This effect is negative, so the level of iron in the magnetized poly(ethyl acrylate-co-divinylbenzene) increased with lower concentration of DVB in the reaction medium. This indicates that greater crosslinking of the polymer tends to reduce the incorporation of iron in the matrix (Figure 5).

The second most important factor is the synergy of the $\mathrm{AD}$ factors, i.e., the associated action of the suspension agent (PVA) and heptane as diluent. This has a negative effect, so the content of iron in the matrix increases with decreasing quantity of PVA and substitution of heptane by toluene for the polymerization.

According to the p-value, only these two effects are significant, because the values are below 0.05 . Table 5 reports the percentage contribution of each of the factors, with highlight on the factors $\mathrm{B}$ and $\mathrm{AD}$ with $26.27 \%$ and $16.37 \%$, respectively.

\subsection{Magnetic force}

The magnetic force values exerted by the poly(ethyl acrylate-co-divinylbenzene) when exposed to a magnetic field are presented in Table 3. From these results, we obtained the Pareto chart shown in the Figure 6.

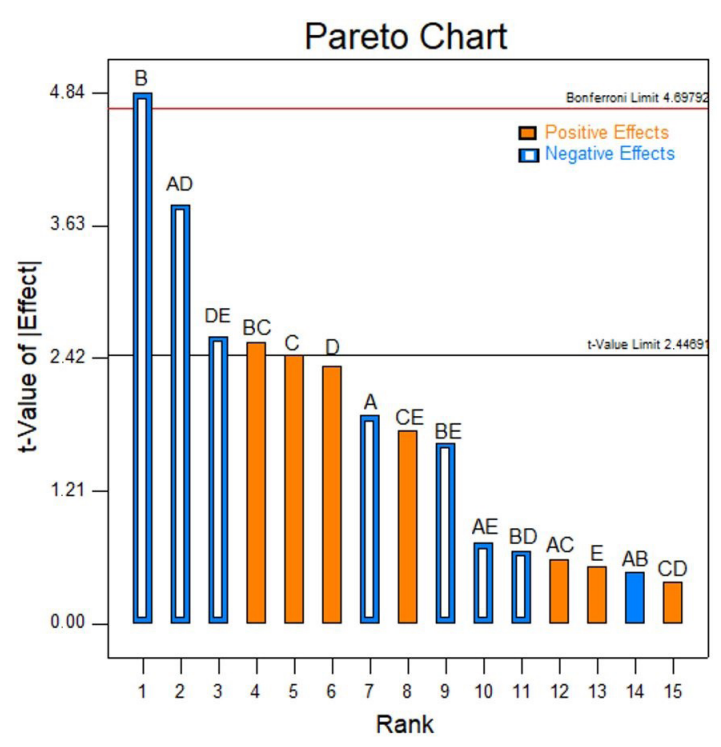

Figure 5. Pareto chart of the iron content incorporated in the poly(ethyl acrylate-co-divinylbenzene).

Table 5. Contribution percentages of the effects that influence the content of iron incorporated in the resins.

\begin{tabular}{lccc}
\hline \multicolumn{1}{c}{ Factor } & Effect & \% Contribution & P-value* $^{*}$ \\
\hline A - \%PVA & -0.25 & 4.03 & 0.2478 \\
B - \%DVB & -0.64 & $\mathbf{2 6 . 2 7}$ & $\mathbf{0 . 0 1 7 1}$ \\
C - \%BPO & 0.33 & 6.70 & 0.1500 \\
D -\%Heptane & 0.31 & 6.17 & 0.1645 \\
E - \%Glycerin & 0.069 & 0.30 & 0.7397 \\
AB & -0.063 & 0.25 & - \\
AC & 0.078 & 0.39 & 0.7048 \\
AD & -0.51 & $\mathbf{1 6 . 3 7}$ & $\mathbf{0 . 0 4 1 9}$ \\
AE & -0.097 & 0.60 & 0.6390 \\
BC & 0.34 & 7.35 & 0.1348 \\
BD & -0.088 & 0.49 & 0.6706 \\
BE & -0.22 & 3.05 & 0.3080 \\
CD & 0.050 & 0.16 & - \\
CE & 0.23 & 3.55 & 0.2804 \\
DE & -0.35 & 7.89 & 0.1272 \\
\hline
\end{tabular}

*P-value $=\mathrm{P}$-value is considered a reliability index of the model obtained, thus p-values lower than 0.01 are considered very reliable, while $0.01 \leq \mathrm{p}<0.05$ indicates moderate reliability, $0.05 \leq \mathrm{p}<0.10$ denotes weak reliability and $\mathrm{p} \geq 0.10$ is considered not significant.

It can be observed that the factor with the greatest influence on the magnetic force of this polymer is the quantity of divinylbenzene (B), with a negative effect, meaning that the magnetic force of the poly(ethyl acrylate-co-divinylbenzene) increases with decrease of the DVB concentration used for the polymerization. This occurs because a larger quantity of the crosslinker increases the number of crosslinks, diminishing the incorporation of iron inside the polymer matrix, and hence its magnetic force.

The second most important factor for this parameter is the AE synergy, i.e., the associated action between the suspension agent PVA and the glycerin added in the continuous medium. This synergistic effect is negative regarding the 


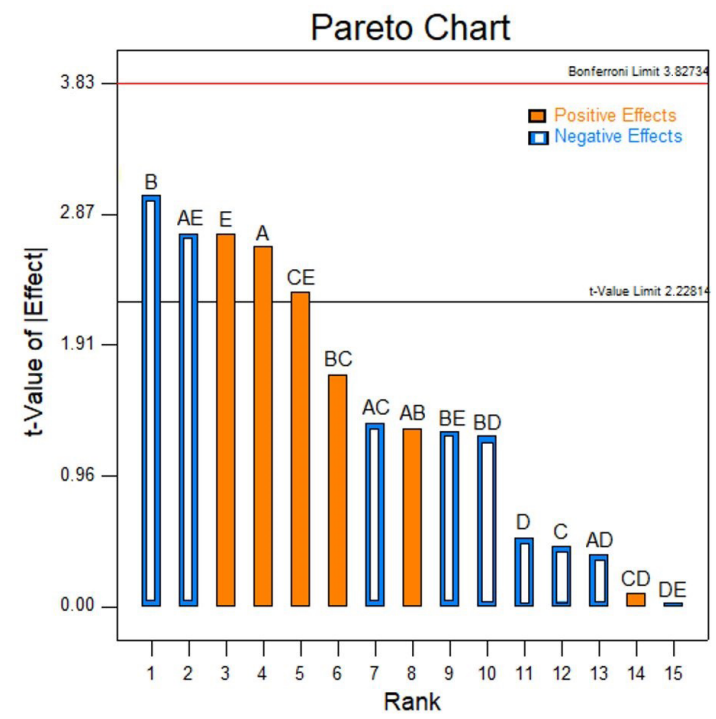

Figure 6. Pareto chart of the magnetic force exerted by the magnetized poly(ethyl acrylate-co-divinylbenzene).

resin's magnetic force, so a higher content of PVA as the aqueous phase containing glycerin causes a reduction of the magnetic force. When this situation occurs, the viscosity of the reaction medium increases considerably, since the PVA also acts as a thickener, probably exceeding the ideal value for good incorporation of iron, thus increasing the magnetic force.

However, when the factors PVA and continuous medium are not associated, they have important positive effects. In this way, the third most relevant factor for the magnetic force is the content of glycerin in the aqueous phase. This occurs because the viscosity of the reaction medium increases, facilitating incorporation of iron in the matrix, and consequently increasing the magnetic force, but without exceeding the ideal level. Factor A (PVA) appears in fourth place when the continuous medium is composed of water alone, which also raises the viscosity of the reaction medium and favors the increase of the magnetic force.

Another important factor is the CE synergy, i.e., the combined action of the polymerization initiator BPO (C) and the presence of glycerin (E) in the continuous medium, acting with a positive effect. Therefore, the magnetic force of the poly(ethyl acrylate-co-divinylbenzene) increases when it is produced with faster reactions and in a more viscous medium.

The factors $\mathrm{B}, \mathrm{AE}, \mathrm{E}, \mathrm{A}$ and $\mathrm{CE}$ have the greatest contribution percentages for the magnetic force parameter, as reported in Table 6 , at $16.16 \%, 13.34 \%, 13.30 \%, 12.39 \%$ and $9.44 \%$, respectively.

The p-values indicate that the factors $\mathrm{B}, \mathrm{AE}, \mathrm{E}$ and $\mathrm{A}$ are highly significant, with chances of being correct of $98.0 \%$, $96.95 \%, 96.92 \%$ and $96.44 \%$. The synergistic factor CE has significance considered reasonable, with $\mathrm{p}$-value above 0.050 , meaning a $94.05 \%$ chance of being the real value, and also of being considered true for the results obtained in the magnetic force analyses.
Table 6. Contribution percentages of the effects that influence the magnetic force of the resins.

\begin{tabular}{lccc}
\hline \multicolumn{1}{c}{ Factor } & Effect & \% Contribution & P-value* \\
\hline A - \%PVA & 4.53 & $\mathbf{1 2 . 3 9}$ & $\mathbf{0 . 0 3 5 6}$ \\
B - \%DVB & -5.18 & $\mathbf{1 6 . 1 6}$ & $\mathbf{0 . 0 2 0 0}$ \\
C - \%BPO & -0.76 & 0.34 & - \\
D -\%Heptane & -0.87 & 0.45 & - \\
E - \%Glycerin & 4.70 & $\mathbf{1 3 . 3 0}$ & $\mathbf{0 . 0 3 0 8}$ \\
AB & 2.23 & 3.01 & 0.2547 \\
AC & -2.31 & 3.21 & 0.2402 \\
AD & -0.66 & 0.26 & - \\
AE & -4.70 & $\mathbf{1 3 . 3 4}$ & $\mathbf{0 . 0 3 0 5}$ \\
BC & 2.92 & 5.13 & 0.1466 \\
BD & -2.15 & 2.80 & 0.2709 \\
BE & -2.21 & 2.95 & 0.2592 \\
CD & 0.16 & 0.016 & - \\
CE & 3.95 & 9.44 & $\mathbf{0 . 0 5 9 5}$ \\
DE & -0.048 & $1.361 \mathrm{E}-003$ & - \\
\hline
\end{tabular}

*P-value $=\mathrm{P}$-value is considered a reliability index of the model obtained, thus $\mathrm{p}$-values lower than 0.01 are considered very reliable, while $0.01 \leq \mathrm{p}<0.05$ indicates moderate reliability, $0.05 \leq \mathrm{p}<0.10$ denotes weak reliability and $p \geq 0.10$ is considered not significant.

\section{Conclusions}

The results show that the quantity of the polymerization initiator BPO is the most important factor for the yield of poly(ethyl acrylate-co-divinylbenzene), since it attained yield greater than $90 \%$ when the BPO concentrations were highest. The quantity of divinylbenzene presented a negative effect in relation to the magnetic force of the resins obtained, and was the main factor found in this work in relation to both responses. Therefore, it was possible to choose the best conditions to obtain magnetized resins with use of the factorial design developed, from the resin denoted as R3, i.e., $0.5 \%$ PVA, $20 \%$ DVB, $10 \% \mathrm{BPO}, 100 \%$ heptane and $50 \%$ glycerin, due to the high yield, high iron content and strong magnetic force.

The experimental conditions of the reaction $\mathrm{R} 3$ can be used as reference to optimize the magnetization process of poly(ethyl acrylate-co-divinylbenzene) resin in terms of the most important factors.

\section{Acknowledgements}

To IFRJ, Nilópolis Campus, and CNPq for financing.

\section{References}

1. Costa, R. C., \& Souza, F. G., Jr. (2014). Preparo de nanocompósitos de maghemita e polianilina assistido por ultrassom. Polimeros: Ciência e Tecnologia, 24(2), 243-249. http://dx.doi.org/10.4322/ polimeros.2014.035.

2. Castanharo, J. A., Ferreira, I. L. M., Costa, M. A. S., Silva, M. R., Costa, G. M., \& Oliveira, M. G. (2015). Microesferas magnéticas à base de poli(metacrilato de metila-co-divinilbenzeno) obtidas por polimerização em suspensão. Polímeros: Ciência e Tecnologia, 25(2), 192-199. http://dx.doi.org/10.1590/01041428.1666 .

3. Mano, E. B., \& Mendes, L. C. (1999). Introdução a polímeros. São Paulo: Blucher. 
4. Cunico, M. W. M., Cunico, M. M., Miguel, O. G., Zawadzki, S. F., Peralta-Zamora, P., \& Volpato, N. (2008). Planejamento fatorial: uma estatística valiosa para a definição de parâmetros experimentais empregados na pesquisa científica. Visão Acadêmica, 9(1), 23-32. http://dx.doi.org/10.5380/acd.v9i1.14635.

5. Pereira, E. R., Fo. (2015). Planejamento fatorial em química: maximizando a obtenção de resultados. São Carlos: EdUFSCar.

6. Costa, C. N., Costa, M. A. S., Maria, L. C. S., Silva, M. R., Souza, F. G., Jr., \& Michel, R. (2012). Síntese e caracterização de copolímeros à base de metacrilato de metila e divinilbenzeno com propriedades magnéticas. Polimeros: Ciência e Tecnologia, 22(3), 260-266. http://dx.doi.org/10.1590/S0104-14282012005000042.

7. Tai, Y., Wang, L., Gao, J., Amer, W. A., Ding, W., \& Yu, H. (2011). Synthesis of $\mathrm{Fe}_{3} \mathrm{O}_{4} @$ poly(methylmethacrylate-codivinylbenzene) magnetic porous microspheres and their application in the separation of phenol from aqueous solutions. Journal of Colloid and Interface Science, 360(2), 731-738. http://dx.doi.org/10.1016/j.jcis.2011.04.096. PMid:21601864.

8. Lan, F., Liu, K.-X., Jiang, W., Zeng, X.-B., Wu, Y., \& Gu, Z.-W. (2011). Facile synthesis of monodisperse superparamagnetic $\mathrm{Fe}_{3} \mathrm{O}_{4} /$ PMMA composite nanospheres with high magnetization. Nanotechnology, 22(22), 225604. http://dx.doi.org/10.1088/09574484/22/22/225604. PMid:21454944.

9. Chen, M., Lin, Z., \& Qian, H. (2008). Preparation of thiophilic paramagnetic adsorbent for separation of antibodies. Chinese Chemical Letters, 19(12), 1495-1498. http://dx.doi.org/10.1016/j. cclet.2008.09.050.
10. Lins, B. F. E. (1993). Ferramentas básicas da qualidade. Ciência da Informação, 22(2), 181-185. http://dx.doi.org/10.18225/ ci.inf.v22i2.502.

11. Paes, A. T. (1998). Itens essenciais em bioestatística. Arquivos Brasileiros de Cardiologia, 71(4), 575-580. http://dx.doi. org/10.1590/S0066-782X1998001000003. PMid:10347932.

12. Reis, M. M. (2017). Conceitos elementares de estatística. Florianópolis: Departamento de Informática e Estatística, Universidade Federal de Santa Catarina. Retrieved in 2017, July 11, from http://www.inf.ufsc.br/ marcelo.menezes.reis/ intro.html.0020

13. Arsham, H. (1988). Kuiper's P-value as a measuring tool and decision procedure for the goodness-of-fit test. Journal of Applied Statistics, 15(2), 131-135. http://dx.doi. org/10.1080/02664768800000020.

14. Silverstein, R. M., \& Webster, F. X. (2000). Identificação espectrométrica de compostos orgânicos (6. ed.). Rio de Janeiro: Livros Técnicos e Científicos.

15. Coutinho, F. M. B., \& Oliveira, C. M. F. (2006). Reações de polymerization em cadeia: mecanismo e cinética. Rio de Janeiro: Interciência.

Received: June 04, 2018

Revised: Sept. 13, 2018

Accepted: Dec. 10, 2018 